\title{
Haunting History. For a Deconstructive Approach to the Past. Por Ethan Kleinberg. Stanford: Stanford University Press, 20I7, I89 p.
}

\author{
Daniel Medel Barragán \\ Universidad Iberoamericana \\ danielle.meursault@gmail.com \\ do \\ DOI: I0.2490I/rehs.v40i157.6I9
}

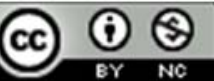

Haunting History. For a Deconstructive Approach to the Past. Por Ethan Kleinberg. Stanford: Stanford University Press, 2017, I89 p. por Daniel Medel Barragán se distribuye bajo una Licencia Creative Commons Atribución-NoComercial 4.0 Internacional.

En medio de un contexto intelectual que intentó "conjurar" al espectro de la deconstrucción, el reciente libro de Ethan Kleinberg -editor de History and Theory-Haunting History. For a Deconstructive Approach to the Past, surge como una interesante provocación que irrumpe y plantea, a nuestra consideración, dos líneas de fuga desde la actualidad historiográfica: a) Las relaciones del historiador con el "pasado"; y, b) La postulación historiográfica del "pasado" en función de sus prácticas al interior de un tipo específico e histórico de saber y, por consiguiente, contingente en sus métodos u operaciones.

Ambas ponen en cuestión lo que Kleinberg denomina "realismo ontológico", esto es la postura comprometida con la actitud de trabajo que asigna a la operación historiográfica una labor de identificar a los acontecimientos y procesos en coordenadas localizables -fijas e inmutables-. Una primera versión de este tipo de realismo puede encontrarse en la actitud historiográfica que opera con la convicción del acceso pleno al pasado. Esta versión "fuerte" convive, en el suelo común de la historiografía actual, con la versión "débil" que asume el carácter de incompletitud epistemológica en los métodos y estrategias de lectura empleados por el historiador. No obstante, afirma Kleinberg, ambas actitudes continúan guiando su actuar metodológico por la "certeza ontológica" de obtener el pasado, es decir, localizarlo y fijarlo.

A partir de la denuncia sobre el realismo ontológico -inconsciente- de la historiografía, la serie de reflexiones -publicadas anteriormente en History and Theory de las que Haunting History... se nutre en forma de libro- encaminarán sus esfuerzos a proponer una forma de relación con el pasado distinta a la planteada por el realismo y el verbo ser del pasado. Los cinco capítulos que componen el libro pueden entonces leerse desde la pluralidad de tópicas, intereses y actitudes 
simultáneas en el quehacer historiográfico contemporáneo. Una de ellas consiste en observar los límites propios de las perspectivas con las cuales intentamos "acceder" al pasado. Dado que éstas se encuentran insertas en un plano de historicidad que les hace contingentes -es decir, temporales y no necesarias-. Es en realidad el historiador y sus operaciones quienes proveen de características ontológicas al pasado, es decir, confieren de un "ser" al mismo.

La "historia de fantasmas" o "historia inquietante" de la deconstrucción es el primer capítulo de Haunting History. En términos de una historia intelectual comprendida a través de momentos específicos (el giro lingüístico, el giro cultural, el fin de la historia y los acontecimientos globales del 9/II) la recepción de la deconstrucción tiene distintos capítulos en función de los contextos y redes intelectuales que la hospedaron. Los lectores de la denominada "French Theory" podrán reconocer dos de los casos más conocidos en el recorrido polémico de la deconstrucción: por un lado, la apertura de instituciones universitarias como Yale, bajo la mano de Paul de Man, a los planteamientos de Jacques Derrida y, por otro, las críticas provenientes del campo historiográfico estadounidense en la década de 1960, que generalmente asociaron a la deconstrucción con los términos de "posmodernismo", "postestructuralismo" o "giro lingüístico". . Y es que, a decir de Kleinberg, el campo historiográfico norteamericano emprendió un largo camino en búsqueda de "conjurar" al fantasma de la deconstrucción mediante argumentos que intentaron "defender" la entereza disciplinar del propio campo. En medio de esta serie de prácticas, la historiografía se escindió entre lo que John G.A. Pocock (partícipe externo de la discusión) denominó metafóricamente Escila y Caribdis; división que incluía a los historiadores enzarzados en la ardua tarea metahistórica conforme a las recientes tendencias teóricas del momento, en detrimento de la labor de establecer procesos y acontecimientos. En el fondo, la incomodidad y acoso del fantasma deconstructivo en el campo historiográfico se debía al acto de evidenciar tanto el orden que subyace a los argumentos como el papel de la imaginación en la reconstrucción histórica.

El segundo capítulo de Haunting History nos exige pensar en torno a la oposición binaria presencia/ausencia. La crítica de Kleinberg dirige entonces sus baterías hacia el paradigma de la "presencia", cuyos exponentes son Hans Ulrich Gumbrecht, Frank R. Ankersmit y Eelco Runia. A grandes rasgos, la recuperación del concepto de presencia implica un desplazamiento desde lo hermenéutico (la "cultura de interpretación" en la historiografía) hacia la experiencia. El concepto de experiencia resultaría central para comprender cómo el pasado puede "visitar" o tocar el presente -los espacios o procesos paralelos, por ejemplo-. En palabras de Kleinberg, el concepto de presencia "ofrece un retorno a lo real que puede ayudarnos a rehabilitar nuestra creencia en el significado, y así esto hace que literalmente traigamos el pasado dentro del presente". . La presencia nos tranquiliza al obtener un referente estable tanto en el tiempo como en el "significado". El concepto de tiempo que maneja el paradigma de la presencia puede ser entendido de la siguiente forma: "el pasado es entendido como lo que ha sido presente, y el futuro como lo que será presente". Acceder al tiempo depende entonces del significado que "fluye" desde una posición espacial fija: la del sujeto (el historiador, por ejemplo).

El privilegio de la presencia, blanco de la crítica deconstructiva, nos permitiría entender al presente a partir de "principios de identidad" y derivar tiempos históricos (pasado/futuro) sin 
comprender el carácter "transhistórico" del mismo: esto, para Kleinberg, resulta crítico al momento de que la presencia "forcluye" la posibilidad de un cambio y "justifica" el estatus de los compromisos desde los cuales se produce un acceso inmediato al pasado "vía el retorno de lo real".

El tercer capítulo nos introduce en una lectura crítica, a través de Dilthey, Droysen y Chladenius de los mecanismos teológico-históricos que han "permanecido" o "reactualizado" a partir de los compromisos con el realismo ontológico. En la opinión de Kleinberg, esta "vía" o modo de trabajo ha sido empleado de "forma silenciosa" para "fijar" el pasado y, de este modo, convertirlo en un elemento accesible en el presente del historiador, marcado por jerarquías que son constantemente restablecidas.

Llegados al cuarto capítulo de Haunting History podemos observar parte de la operatividad que la crítica de Kleinberg tiene al interior de los contextos historiográficos compuestos por convenciones disciplinares. El caso particular que el autor pone a nuestra consideración es el de los departamentos de historia estadounidenses. A partir de este momento el debate se enfoca en el sentido común que, bajo la égida del realismo ontológico, afirma la muerte de la "historia teórica". De este modo, aun cuando la disciplina histórica no es "inocente" en sus aproximaciones al pasado, existe un trasfondo ontológico que ve en el pasado un elemento "preexistente" en sí mismo.

El problema surge al momento del denominado "límite analógico" o cómo las distintas prácticas refuerzan y reproducen los aspectos normativos y disciplinares en la historia. Resulta entonces que el límite analógico funciona como un dispositivo que justifica el uso del realismo ontológico, no sólo como una vía preferencial de acceso al pasado, sino como la única vía de pensar y reflexionar respecto de nuestras relaciones con el pasado.

Incluso, para Kleinberg, el realismo ontológico ha llegado a condicionar las formas materiales de transmisión del conocimiento histórico. Esto resulta vital para problematizar las perspectivas que observan en la materialidad de la escritura una condición para la recepción y difusión de la historia. Al mismo tiempo, nos emplaza en el problema de "introducir" formas nuevas de transmisión -el ebook- al paradigma del realismo ontológico: esto es, "innovar" los medios o la infraestructura (las humanidades digitales, por ejemplo) en las "nuevas formas textuales" -a decir de Jonathan Sterne- sin "innovar" o desplazar al realismo ontológico como matriz normativa. Ambos elementos resultarían incompatibles en virtud de la forma teleológica que el realismo mantiene en una forma lineal de escritura (la materialidad como mero soporte de las ideas o estrategias narrativas lineales) para las comunicaciones historiográficas.

Y es que, a decir de Kleinberg, el pasado por sí solo no posee propiedades ontológicas e, incluso, "si lo hace, tiene una ontología latente", de tal modo que "el evento pasado no puede ser hecho presente". ${ }^{3}$ Es en el capítulo final donde hace su "aparición" la recuperación derrideana del autor: el pasado como una hantologie; bajo una forma no emplazable en los parámetros ontológicos de la presencia o ausencia y, por consiguiente, situada en una relación no jerárquica donde la inestabilidad de ambos polos se asume desde la incomodidad provocada por el asedio de un 
pasado espectral. El pasado "sería" indeterminable en la medida que no podemos reconstruir un "original", una identidad que no existió. En este sentido, el realismo ontológico perdió su capacidad tranquilizante de ser una vía al pasado debido a las innovaciones tecnológicas que habrían afectado sus vínculos con las prácticas de publicación. La relación planteada por Kleinberg se distingue de otras al mantener un espacio de "apertura" a las irrupciones de elementos latentes del pasado en el presente. El asedio implica que, a pesar de las extensas interpretaciones en búsqueda de una "clausura" o "cierre" de las lecturas historiográficas, siempre existirá la latencia incómoda del pasado que permite y condiciona la escritura de la historia bajo nuevas aproximaciones. El "pasado que es" postulado por Kleinberg se entiende tanto como una teoría donde distintas y múltiples temporalidades "convergen" o pueden convivir en un presente, el nuestro. El pasado ausente, a la manera de un fantasma que nos asedia o puede asediarnos en cualquier momento, no posee cualidades ontológicas plenas: tiene, en todo caso, una ontología latente que siempre se encuentra en "posibilidad" y contingencia. Cuando un pasado latente retorna, la historia es asediada y nos sitúa frente a un pasado en constante "polisemia" y "contradicción". En el rango de análisis orientado al "contexto presente" del historiador (las convenciones e instituciones que albergan la práctica historiográfica), la utilidad de la lectura particular de Kleinberg radica entonces en la posibilidad de denunciar las jerarquías conceptuales que ordenan la escritura de la historia junto al instante de "decisión" del historiador que privilegia, de acuerdo con una serie de compromisos de carácter agónico, un pasado particular de entre muchos otros. Estos otros, señala Kleinberg, son puestos en duda o desplazados mediante términos como "inconcebibles", "inimaginables" o "imposibles". Ante una historiografía con mayores índices de reflexividad respecto de sus presupuestos y condiciones de posibilidad, la táctica deconstructiva elaborada por Haunting History nos revela cómo podemos entender el campo de tensiones y fuerzas en constante conflicto por las jerarquía de los significados entendidos como condición de posibilidad en la escritura de la historia: la capacidad de leer fuera de contexto (iterabilidad). Nos muestra los modos y estrategias necesarios en la legitimidad de nuestros relatos y los procesos controversiales donde las lecturas deconstructivas nos muestran las jerarquías conceptuales o binarias -realidad/ficción, por ejemplo- que ordenan las interpretaciones y relaciones con un pasado "donde nunca hemos estado". ${ }^{4}$ De este modo, la propuesta crítica de Kleinberg intenta escapar de lo que puede denominarse "el concepto metafísico de historia", es decir, la idea de una historia lineal (teleológica, continua o ligada a una escatología) donde la relación con el pasado se estructure como una continua acumulación de significados y, en suma, "clausure" el exceso de sentido y las diferencias del pasado en las interpretaciones del mismo. Al mismo tiempo, la aproximación deconstructiva al pasado que coloca en contingencia a las operaciones historiográficas y sus coordenadas binarias tiene la virtud de recordar la constitución polémica y política de la historia. Pero podemos ir más allá al recordar la capacidad del historiador -en su vita activa- de "desestabilizar" los relatos autorizados, situar en perspectiva histórica los pasados hegemónicos y mostrar el pasado en un sentido abierto, esto es crítico del presentismo.

\section{Notas}

I En este sentido, son ejemplares las críticas de Richard J. Evans (In Defense of History), Carolyn Steedman Jones (Fever of Archive) e, incluso, en un primer momento, de Dominick LaCapra y 
Hayden White. Ethan Kleinberg. Haunting History. For a Deconstructive Approach to the Past (Stanford: Stanford University Press, 2017), I3.

2 Kleinberg, Haunting History, 59.

3 Kleinberg, Haunting History, 2.

4 El autor identifica otras oposiciones como presencia/ausencia, estable/relativo, posmoderno/tradicional. 